\title{
Influence of quantum well and barrier composition on the spectral behavior of InGaAs quantum dots-in-a-well infrared photodetectors
}

\author{
G. Jolley, ${ }^{a}$ L. Fu, H. H. Tan, and C. Jagadish \\ Department of Electronic Materials Engineering, Research School of Physical Sciences and Engineering, \\ The Australian National University, Canberra, Australian Capital Territory 0200, Australia
}

(Received 17 August 2007; accepted 5 October 2007; published online 25 October 2007)

\begin{abstract}
We report on the spectral behavior of two different quantum dots-in-a-well infrared photodetectors grown by low-pressure metal-organic chemical vapor deposition. $\operatorname{In}_{0.5} \mathrm{Ga}_{0.5} \mathrm{As}$ quantum dots embedded in an $\mathrm{In}_{0.15} \mathrm{Ga}_{0.85} \mathrm{As} / \mathrm{GaAs}$ quantum well $(\mathrm{QW})$ or a $\mathrm{GaAs} / \mathrm{Al}_{0.2} \mathrm{Ga}_{0.8} \mathrm{As} \mathrm{QW}$ have been incorporated into photodetectors and were characterized. A spectral response in the 3-5 $\mu \mathrm{m}$ atmospheric window has been achieved by adopting the GaAs/ $\mathrm{Al}_{0.2} \mathrm{Ga}_{0.8} \mathrm{As} \mathrm{QW}$. (C) 2007 American Institute of Physics. [DOI: 10.1063/1.2802559]
\end{abstract}

Quantum dots (QDs) are attracting a lot of research interest both due to their unique physical properties and their potential use in superior optoelectronic devices. Theoretical studies have suggested that quantum dot infrared photodetectors (QDIPs) can outperform quantum well infrared photodetectors (QWIPs). ${ }^{1,2}$

QDs are routinely grown in the self-assembled StranskiKrastanow growth mode. Due to the random nature of the self-assembled growth process, controlling the electronic structure and optical quality of the QDs is a difficult task. The growth parameters that lead to high optical quality QDs (high density, uniform size, and free from defects) do not necessarily result in QDs with an optimized band structure from a device performance point of view. The quantum dotsin-a-well (DWELL) structure where QDs are embedded in a quantum well, which in turn is sandwiched between barrier layers, has been proposed as a way of tailoring the electronic properties of QDs without altering the QD growth parameters. ${ }^{3}$ Therefore, the optical and electronic properties of the QDs can be independently optimized.

The majority of QDIPs reported in the literature have been grown by molecular-beam epitaxy (MBE). For the general InGaAs/GaAs QDIP system, with or without wells, spectral responses within the medium- and long-wavelength infrared bands have been reported. ${ }^{4,5}$ However, for the very few reports of MOCVD growth ${ }^{6,7}$ there are no known reports of DWELL structures that are similar to the detectors reported here.

In this letter we report on two different DWELL photodetectors grown by metal-organic chemical vapor deposition (MOCVD), $\quad \operatorname{In}_{0.5} \mathrm{Ga}_{0.5} \mathrm{As} / \mathrm{In}_{0.15} \mathrm{Ga}_{0.85} \mathrm{As} / \mathrm{GaAs}$ and $\mathrm{In}_{0.5} \mathrm{Ga}_{0.5} \mathrm{As} / \mathrm{GaAs} / \mathrm{Al}_{0.2} \mathrm{Ga}_{0.8} \mathrm{As}$, hereafter referred to as GaAs and AlGaAs QDIPs, respectively.

The MOCVD growth parameters for $\mathrm{In}_{0.5} \mathrm{Ga}_{0.5}$ As QDs grown directly on GaAs without QWs have previously been optimized $^{8}$ and incorporated into QDIPs. ${ }^{9}$ However, the range over which the spectral peak that these photodetectors can be tuned is limited and outside of the technologically useful atmospheric window bands. In this work, a strong spectral peak within the $3-5 \mu \mathrm{m}$ band is achieved due to the band structure engineering of the AlGaAs DWELL structure.

The GaAs and AlGaAs DWELL structures investigated are depicted in Figs. 1(a) and 1(b). All growths were per-

${ }^{a)}$ Electronic mail: gvj109@rsphysse.anu.edu.au formed using low pressure MOCVD on (001) oriented semiinsulating (SI) GaAs substrates. When barrier material is grown at a low temperature, it results in poor quality material, so, as much as possible, it was grown at a temperature higher than the QD growth temperature. The case of GaAs growth at $600{ }^{\circ} \mathrm{C}$ results in good quality material, whereas the $\mathrm{AlGaAs}$ barriers required a growth temperature of $650{ }^{\circ} \mathrm{C}$. After the initial amount of barrier material required to cap the QDs is deposited at the QD growth temperature, the growth is interrupted while the temperature is ramped, then the remainder of the barrier layer is deposited. Wafers were processed into $250 \mu \mathrm{m}$ square mesastructures using standard lithography and wet chemical etching. $150 \mu \mathrm{m}$ circular top contacts and bottom contacts were formed by e-beam evaporation of $\mathrm{Ge} / \mathrm{Ni} / \mathrm{Au}$ followed by a $60 \mathrm{~s}$ alloying time at $400{ }^{\circ} \mathrm{C}$. Devices were then wire bonded into DIP packages and mounted into a liquid nitrogen cooled dewar for characterization.

From plan-view transmission electron microscopy (TEM) images of single QD layers (not shown) grown under the same conditions as the two QDIP structures, the QD densities of the GaAs and AlGaAs detectors are estimated to be $4 \times 10^{10}$ and $2 \times 10^{10} \mathrm{~cm}^{-2}$, respectively. TEM measurements indicate that in both cases, the average QD base width is $\sim 20 \mathrm{~nm}$ with a height of $\sim 5 \mathrm{~nm}$.

Room temperature and $77 \mathrm{~K}$ photoluminescence (PL) measurements were performed using the $532 \mathrm{~nm}$ line of a frequency doubled diode pumped solid state laser. The PL is detected by an InGaAs photodetector after being dispersed through a $0.5 \mathrm{~m}$ monochromator. A strong room temperature PL signal was detected from the structures indicating that high quality defect-free QDs have been grown. The energies of the $77 \mathrm{~K}$ PL peak of the GaAs and the AlGaAs detectors are $1.115 \mathrm{eV}$ [full width at half maximum (FWHM) of $72 \mathrm{meV}$ ] and $1.207 \mathrm{eV}$ (FWHM of $65 \mathrm{meV}$ ), respectively. The blueshifting of the AlGaAs detector PL is partly due to the interdiffusion ${ }^{10}$ that occurs between the QD and the QW material when the barrier material is grown at $650{ }^{\circ} \mathrm{C}$.

The normal incidence spectral response was measured with a Nicolet Impact 400 Fourier transform infrared spectrometer and a SRS 570 low-noise current preamplifier. The $77 \mathrm{~K}$ voltage dependent spectral response of the GaAs device is shown in Fig. 2. As has been noted previously in experimental and theoretical studies, ${ }^{11}$ due to the large base to height ratio of the QDs, the absorption coefficients for 


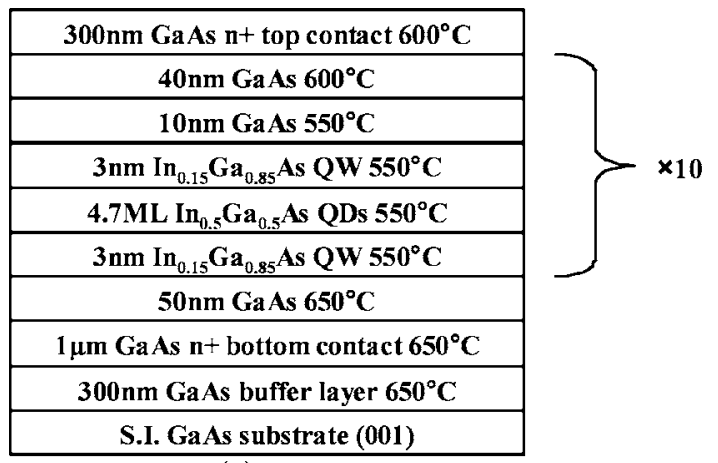

(a)

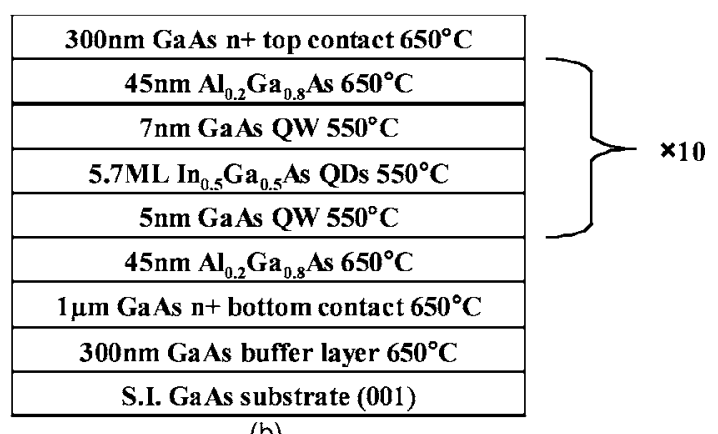

(b)

FIG. 1. Schematic diagram of the dots-in-a-well (DWELL) structures: (a) GaAs and (b) AlGaAs.

in-plane and $z$-polarized light are greatest at different regions of the IR band. For the sort of QDs presented here, strong absorption of in-plane polarized light typically occurs in the far infrared region at about $20 \mu \mathrm{m}$. Therefore, the transitions responsible for the photocurrent observed for the devices studied here are due to quantization changes in the $z$ direction. For such transitions, normal incident radiation is expected to have a weak interaction. Addition absorption can occur due to unintentional scattering of in-plane polarized light into the $z$ direction.

For a DWELL detector only operating on bound-tocontinuum transitions, the shape of the spectral response curve is not expected to have a significant dependence on the bias voltage since all electrons are excited to a similar state in the continuum and, therefore, have nearly identical chances of escaping the QDs and contributing to the photocurrent. From the lack of voltage dependence on the spectral response and the large FWHM of $42 \mathrm{meV}$ (at the bias of $1.0 \mathrm{~V})$, it is evident that the photoresponse is due to boundto-continuum transitions.

A number of GaAs detectors with different well widths within a $5-7 \mathrm{~nm}$ range were fabricated; the energy of the spectral peaks were all observed to have a variation of only less than $1 \%$. Since the GaAs detectors are operating on bound-to-continuum transitions, the spectral response is expected to have only a weak dependence on the well width. The ground-state wavefunction is mostly confined to the QD and, therefore, varying the well width within the 5-7 nm range is going to have only a very small influence on the ground-state energy. This has been confirmed by PL measurements which revealed an electron-heavy hole transition energy variation of less than $2 \%$. Also, the continuum band edge in the vicinity of the QD is weakly influenced by the well so the bound-to-continuum energy should be weakly dependent on the well width.

Without adversely affecting the optical quality of the QDs, it was not possible to alter the QD growth parameters such that the GaAs detectors operated in a bound-to-bound mode, nor was it possible to significantly tailor the spectral peak such that a response occurred in either of the atmospheric windows. Our result is in contrast to reports in the literature of DWELL QDIPs of a similar structure, with spectral responses that have a significant dependence on well width. Krishna has reported spectral tailoring from 7.2 to $11 \mu \mathrm{m}$ for a peak that is attributed to dot-to-well transitions, from an InAs/InGaAs/GaAs detector grown by MBE. ${ }^{12}$ In general, DWELL spectral tailoring is only expected for dotto-well transitions. However, the reduced growth parameter Downloaded 24 Feb 2008 to 150.203 .178 .41 . Redistribution subject window for MOCVD-grown InGaAs QDs limits the control over the location of the excited state and has contributed to the lack of DWELL detector tuning. For a theoretical analysis of the spectral properties of DWELL detectors of this sort, see Ref. 13.

The AlGaAs DWELL has two main advantages over the GaAs DWELL, the wells are lattice matched to the barriers so they can be grown to any width without the adverse effects of strain, and the well to barrier conduction band offset can be larger $\left(0.167 \mathrm{eV}\right.$ for $\left.\mathrm{Al}_{0.2} \mathrm{Ga}_{0.8} \mathrm{As}\right)$.

The AlGaAs detectors were investigated in an effort to obtain a response in the $3-5 \mu \mathrm{m}$ band. Figure 3 shows the spectral response measured at various applied biases. Two distinct peaks in the spectral response are clearly seen. The simplified conduction band diagram shown in Fig. 4 displays the energy levels that are responsible for the spectral response peaks. The peak at about $7 \mu \mathrm{m}\left(E_{2} \sim 178 \mathrm{meV}\right)$ is attributed to transitions between the QD ground state and the first excited state of the combined dot and well systems. Although calculations show that the ground state energy of a $12 \mathrm{~nm} \mathrm{Al}{ }_{0.2} \mathrm{Ga}_{0.8} \mathrm{As} / \mathrm{GaAs} \mathrm{QW}$ is only about $23 \mathrm{meV}$ above the GaAs conduction band edge, the quantum well has a more significant effect on the dot-to-well transition energy since the first excited state of the $\mathrm{QD} / \mathrm{QW}$ is of a higher energy than the QW ground state. The PL peak of the AlGaAs detector is $92 \mathrm{meV}$ blueshifted compared with the GaAs detector indicating that the electrons are less confined to the QDs in the former. However, due to the confinement

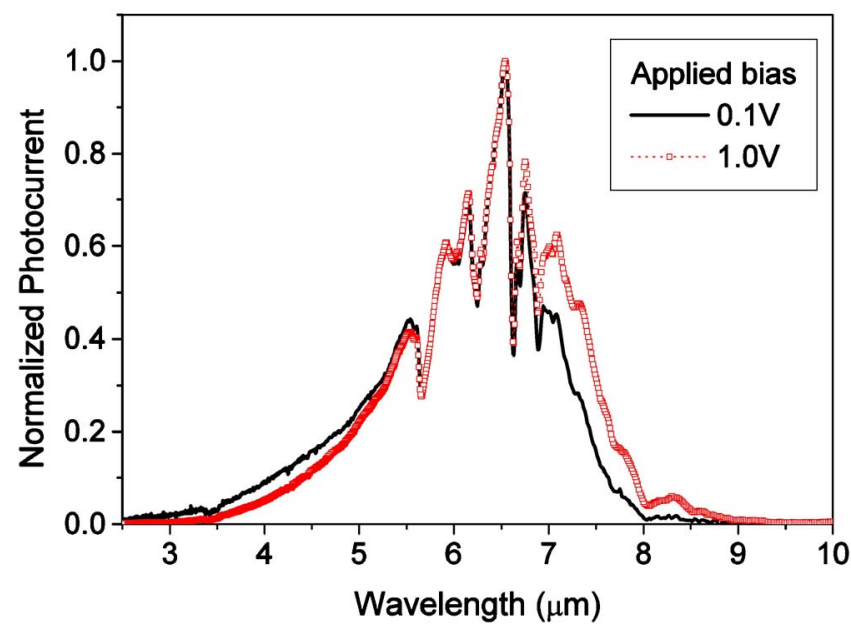

FIG. 2. (Color online) The $77 \mathrm{~K}$ normal incident spectral response of the GaAs detector for two bias voltages.

to AIP license or copyright; see http://apl.aip.org/apl/copyright.jsp 


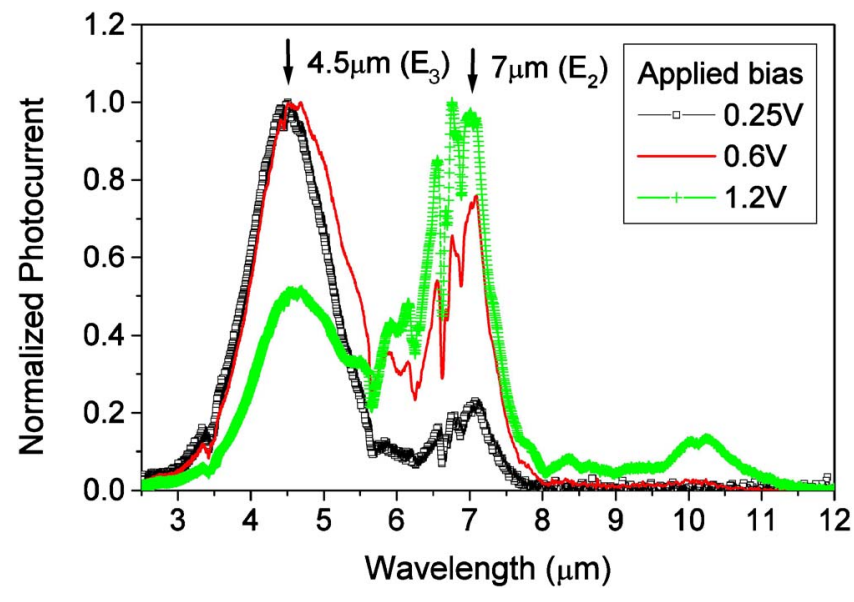

FIG. 3. (Color online) The $77 \mathrm{~K}$ normal incident spectral response of the $\mathrm{AlGaAs}$ detector as a function of applied bias.

energy of the QW, the dot-to-well transition of the AlGaAs detector is of a very similar energy to the dot-to-continuum transition of the GaAs detector $(\sim 190 \mathrm{meV})$.

It is believed that the spectral peak at about $4.5 \mu \mathrm{m}$ $\left(E_{3} \sim 276 \mathrm{meV}\right)$ is due to transitions between the QD ground state and continuum states in the AlGaAs barriers. It is assumed that the energy of this transition at low bias voltages is approximately the amount of energy required to take an electron from the ground state of the QD to the conduction band edge of the AlGaAs barrier. This energy should be equal to the conduction band offset (CBO) of the AlGaAs/GaAs QW plus the confinement energy of the QD relative to the GaAs band edge $\left(E_{\mathrm{QD}}\right)$. The confinement energy of the QD is therefore $E 3-C B O=276-167 \mathrm{meV}$ $=109 \mathrm{meV}$. The energy required to take an electron from the QD ground state to the ground state of the QW is about $23 \mathrm{meV}$ larger $\left(E_{1} \sim 132 \mathrm{meV}\right)$. This energy corresponds closely with the small $10.3 \mu \mathrm{m}$ peak observed at higher bias voltages.

The escape probability and photoelectric gain for a given transition are voltage dependent. This dependence on voltage is different for the dot-to-well and the dot-to-continuum transitions, leading to a voltage dependent spectral response. Characteristically, this type of detector is a voltage tunable two color detector.

Normal incidence responsivity and detectivity measurements were performed using a calibrated $800{ }^{\circ} \mathrm{C}$ blackbody radiation source, chopped at $140 \mathrm{~Hz}$, a Stanford Research Systems SR570 low noise current preamplifier and a Stanford Research Systems SR760 fast Fourier transform spectrum analyzer. Figure 5 shows the peak responsivity and detectivity measured at various biases at $77 \mathrm{~K}$. The peak detectivity of the GaAs detectors is $3.2 \times 10^{9} \mathrm{~cm} \mathrm{~Hz}^{1 / 2} / \mathrm{W}$ and the responsivity at the bias that gives the maximum detectivity is $40 \mathrm{~mA} / \mathrm{W}$. The peak detectivity of the AlGaAs

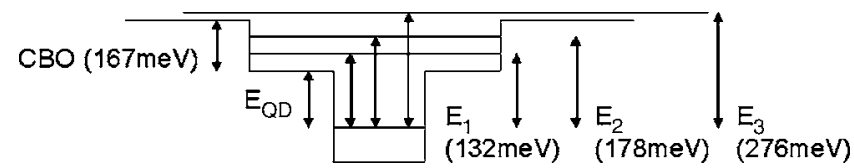

FIG. 4. Simplified schematic diagram of the conduction band of the AlGaAs dots-in-a-well structure.

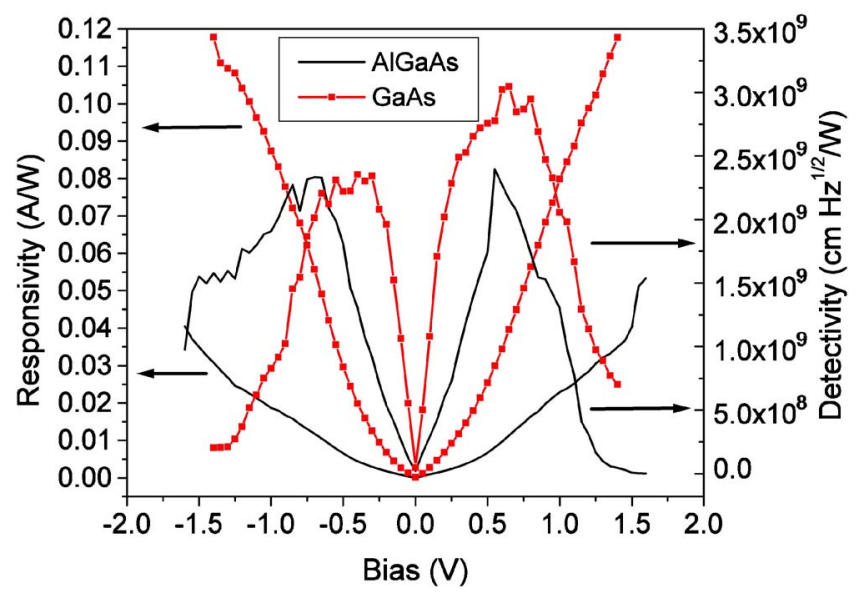

FIG. 5. (Color online) $77 \mathrm{~K}$ peak responsivity and detectivity of the GaAs and $\mathrm{AlGaAs}$ photodetectors as a function of applied bias.

detectors is $2.4 \times 10^{9} \mathrm{~cm} \mathrm{~Hz}^{1 / 2} / \mathrm{W}$ and the responsivity is $8 \mathrm{~mA} / \mathrm{W}$.

The possible contributing factors to the reduced responsivity of the AlGaAs detector are a reduced QD density, larger electron capture probabilities from the AlGaAs barrier layers into the QDs, the reduced electron mobility of the AlGaAs barriers, and different background doping densities of the AlGaAs and GaAs barrier layers. With further optimization of the DWELL parameters and the doping profile of the active region, it is believed that the detectivity and responsivity of the $\mathrm{AlGaAs}$ detector can be further improved.

In summary we have investigated the spectral response of $\operatorname{In}_{0.5} \mathrm{Ga}_{0.5} \mathrm{As} / \mathrm{In}_{0.15} \mathrm{Ga}_{0.85} \mathrm{As} / \mathrm{GaAs}$ and $\mathrm{In}_{0.5} \mathrm{Ga}_{0.5} \mathrm{As} / \mathrm{GaAs} / \mathrm{Al}_{0.2} \mathrm{Ga}_{0.8}$ As DWELL QDIPs grown by low pressure MOCVD. The AlGaAs barriers have been effective in providing states in the continuum at a higher energy, thereby a photoresponse peak at about $4.6 \mu \mathrm{m}$ has been obtained.

Thanks are due to Tom Halstead and Michael Aggett for expert technical advice. The Australian Research Council is gratefully acknowledged for the financial support.

${ }^{1}$ M. Mashade, M. Ashry, and A. Nasr, Semicond. Sci. Technol. 18, 891 (2003).

${ }^{2}$ V. Ryzhii, I. Khmyrova, V. Mitin, M. Stroscio, and M. Willander, Appl. Phys. Lett. 78, 3523 (2001).

${ }^{3}$ S. Raghavan, P. Rotella, A. Stintz, B. Fuchs, S. Krishna, C. Morath, D. A. Cardimona, and S. W. Kennerly, Appl. Phys. Lett. 81, 1369 (2002).

${ }^{4}$ S. Chakrabarti, A. D. Stiff-Roberts, X. H. Su, P. Bhattacharya, G. Ariyawansa, and A. G. U. Perera, J. Phys. D 38, 2135 (2005).

${ }^{5}$ Z. Ye, J. C. Campbell, Z. Chen, E. T. Kim, and A. Madhukar, J. Appl. Phys. 92, 7462 (2002).

${ }^{6}$ J. Szafraniec, S. Tsao, W. Zhang, H. Lim, M. Taguchi, A. A. Quivy, B. Movaghar, and M. Razeghi, Appl. Phys. Lett. 88, 121102 (2006).

${ }^{7}$ M. P. Pires, S. M. Landi, C. V.-B. Tribuzy, L. A. Nunes, E. Marega, and P. L. Souza, J. Cryst. Growth 272, 192 (2004).

${ }^{8}$ P. Lever, "Interdiffusion and MOVPE Growth of Self-Assembled InGaAs QD Structures and Devices." Ph.D. thesis, Australian National University, 2004.

${ }^{9}$ L. Fu, P. Lever, K. Sears, H. H. Tan, and C. Jagadish, IEEE Electron Device Lett. 26, 628 (2005).

${ }^{10}$ R. Leon, Y. Kim, C. Jagadish, M. Gal, J. Zou, and D. J. H. Cockayne, Appl. Phys. Lett. 69, 1888 (1996).

${ }^{11}$ P. Boucaud and S. Sauvage, C. R. Phys. 4, 1133 (2003).

${ }^{12}$ S. Krishna, J. Phys. D 38, 2142 (2005).

${ }^{13}$ N. Vukmirovic, D. Indjin, Z. Ikonic, and P. Harrison, Appl. Phys. Lett. 88, 251107 (2006) 